\section{Becoming an emergency resident}

To the Editor: I read with interest the piece by Dr. McCauley regarding a program director's perspective on selecting residents. ${ }^{1}$ I am happy to see attention being paid to an important topic and with welcome insights for prospective emergency medicine applicants.

In the spirit of "healthy rivalry" alluded to in the article, I feel that clarification and embellishment may be helpful in some key areas.

First, it is important for candidates to realize that selection strategies differ from program to program in important ways. For example, the University of Toronto places less emphasis on reference letters and more on the candidates' personal letters and experiences. ${ }^{2}$ We also do not start over at interview time; scores on the CaRMS (Canadian Residency Matching Service) application are carried over to the final score and thus contribute to the ranking process.

Second, there is a body of literature developing that suggests that properly structured application assessment protocols can generate highly reliable ranking lists..$^{2-4}$ Programs may decide to interview extra candidates based on elective experience or local training. This can easily be justified based on the philosophies of maximizing opportunities for local talent, rewarding those who take the time to visit on elective, or minimizing risk by highly rating those with whom one has personal experience. It should be understood that these candidates are considered in spite of, not because of, objective selection criteria. This may not only reduce the objectivity of the process, but may provide an unfair advantage to those who either have the time and money to travel for elective time, or have been lucky enough to get into the local medical school.

Finally, I agree that the interview provides a program with valuable insights into a candidate's "fit" with the program. I would also suggest, however, that the candidates view the interview as a golden opportunity to determine how well the program fits with them. Again, I congratulate Dr. McCauley on shedding some light on this angst-ridden process.

\section{Glen Bandiera, MD, MEd}

Program Director

FRCP (EM) Residency Program

University of Toronto

Toronto, Ont.

\section{References}

1. McCauley B. Becoming an emergency resident: a program director's perspective. Can J Emerg Med 2004;6(1):53-4.

2. Bandiera GW, Regehr G. Reliability of a structured interview scoring instrument for a Canadian postgraduate emergency medicine training program. Acad Emerg Med 2004;11(1):1 27-32.

3. Aghababian R, Tandberg D, Iserson K, Martin M, Sklar D. Selection of emergency medicine residents. Ann Emerg Med 1993;22(11):1753-61.

4. Bandiera G, Regehr G. A structured application assessment instrument is a reliable tool for assessing applications to Canadian postgraduate training programs in Emergency Medicine. Acad Emerg Med 2003;10:594-8.

\section{Correction}

In the May issue of CJEM, the affiliation of one author of an article ${ }^{1}$ was inadvertently omitted. At the time of writing Dr. Dan Somogyi was with the Department of Family Medicine, University of Pittsburgh, Pittsburgh, Pa.

\section{Reference}

1. Vaillancourt C, Shrier I, Vandal A, Falk M, Rossignol M, Vernec A, et al. Acute compartment syndrome: How long before muscle necrosis occurs? Can J Emerg Med 2004;6(3):147-54.

\section{Missed scientific abstract from the 2004 CAEP/ACMU scientific assembly}

The CAEP research abstract published on this page was inadvertently missed during the compilation of the May issue of CJEM. This abstract was awarded a poster presentation. The Research Committee apologies for any inconvenience this may have caused.

Management of toxic alcohol ingestions after the introduction of Fomepizole

Green RS, Department of Emergency Medicine, Dalhousie University, Halifax, NS

INTRODUCTION: Ethylene glycol and methanol ingestions are relatively uncommon but potentially lethal overdoses. Traditional management of toxic alcohol ingestions is directed at minimizing the generation of toxic metabolites by alcohol dehydrogenase $(\mathrm{ADH})$ with ethanol infusions, and hemodialysis (HD) for toxin removal. Recently, an alternative agent for ADH blockade was introduced into clinical practice. The purpose of this study was to review the management of toxic alcohol ingestions in a tertiary care referral center after the introduction of fomepizole. METHODS: Data was collected on all patents treated for a toxic alcohol ingestion for a 1 year period after the introduction of fomepizole to hospital formulae. Patients were identified by ED, pharmacy, hemodialysis and ICU databases. The patients medical records were reviewed, and data was recorded on a predetermined computerized data collection form.
RESULTS: Overall, twenty one (21) toxic ingestions (14 methanol; 7 ethylene glycol) were identified over the one year period. Fomepizole was used for ADH blockade in 10/21 patients; ETOH infusions in 16/21 patients (combined ETOH and fomepizole use in 5/21). All patients were admitted to an intensive care unit and all received hemodialysis. On preliminary analysis, there was no apparent difference in time from ingestion to ED presenataion, serum $\mathrm{pH}$, serum bicarbonate, or serum creatinine between groups treated with fomepizole or ETOH infusions. In addition, there was no apparent difference in the need for HD, ICU admission, ICU LOS, or total LOS. CONCLUSIONS: Despite theoretical advantages, the addition of fomepizole to the management of toxic alcohol ingestions does not seem to change the need for HD or ICU admission. Further study is needed to determine the role fomepizole in tertiary care centers. 